\title{
Catheter-related giant right atrial thrombosis mimicking a myxoma: A case report
}

\author{
OVIDIU STIRU ${ }^{1,2^{*}}$, RAZVAN DRAGULESCU ${ }^{1}$, ROXANA CARMEN GEANA ${ }^{1}$, ANDREEA CHIBULCUTEAN ${ }^{1}$, \\ LAURA RADUCU ${ }^{3,4}$, ADRIAN TULIN ${ }^{4,5^{*}}$, ANDRA BALCANGIU STROESCU ${ }^{2}$, CAMELIA DIACONU ${ }^{6,7}$, \\ CORNEL SAVU ${ }^{8,9}$, NICOLAE BACALBASA ${ }^{10-12}$, IRINA BALESCU ${ }^{13}$ and VLAD ANTON ILIESCU ${ }^{1,2}$ \\ ${ }^{1}$ Department of Cardiovascular Surgery, 'Prof. Dr. C. C.' Iliescu Emergency Institute for Cardiovascular Diseases, \\ 022322 Bucharest; Departments of ${ }^{2}$ Cardiovascular Surgery, and ${ }^{3}$ Plastic and Reconstructive Microsurgery, \\ 'Carol Davila' University of Medicine and Pharmacy, 020021 Bucharest; ${ }^{4}$ Department of General Surgery, \\ 'Prof. Dr. Agripa Ionescu', Clinical Emergency Hospital, 011356 Bucharest; Departments of ${ }^{5}$ Anatomy, \\ and ${ }^{6}$ Internal Medicine, 'Carol Davila' University of Medicine and Pharmacy, 020021 Bucharest; \\ ${ }^{7}$ Department of Internal Medicine, Clinical Emergency Hospital of Bucharest, 105402 Bucharest; \\ ${ }^{8}$ Department of Thoracic Surgery, 'Carol Davila' University of Medicine and Pharmacy, 020021 Bucharest; \\ ${ }^{9}$ Department of Thoracic Surgery, 'Marius Nasta' National Institute of Pneumology, 050159 Bucharest; \\ ${ }^{10}$ Department of Obstetrics and Gynecology, 'Carol Davila' University of Medicine and Pharmacy, \\ 020021 Bucharest; ${ }^{11}$ Department of Obstetrics and Gynecology, 'I. Cantacuzino' Clinical Hospital, \\ 030167 Bucharest; ${ }^{12}$ Department of Visceral Surgery, Center of Excellence in Translational Medicine, \\ 'Fundeni' Clinical Institute, 022328 Bucharest; ${ }^{13}$ Department of Surgery, \\ 'Ponderas' Academic Hospital, 021188 Bucharest, Romania
}

Received December 31, 2020; Accepted February 2, 2021

DOI: $10.3892 /$ etm.2021.10035

\begin{abstract}
Despite the development of imagistic methods, the differential diagnosis of a right atrial mass may be difficult to be established, the most common pathologies which should be taken in consideration being represented by thrombus, tumors, prominent crista terminalis, or vegetation of infectious endocarditis. In this study, we present the case of a 63-year-old man with chronic kidney disease, in hemodialysis (HD) with
\end{abstract}

Correspondence to: Dr Nicolae Bacalbasa, Department of Obstetrics and Gynecology, 'Carol Davila' University of Medicine and Pharmacy, Eroilor Sanitari Bld no. 8, 020021 Bucharest, Romania

E-mail: nicolae_bacalbasa@yahoo.ro

*Contributed equally

Abbreviations: Ao, aorta; BBAVF, brachial-basilic arteriovenous fistula; CVC, central venous catheter; MRI, magnetic resonance imaging; CRAT, catheter-related right atrial thrombosis; CPB, cardiopulmonary bypass; ECG, electrocardiogram; HD, hemodialysis; LV, left ventricle; RA, right atrium; RAM, right atrial mass; TEE, transesophageal echocardiography; TTE, transthoracic echocardiogram

Key words: hemodialysis, thrombus, myxoma, right atrial mass, right atriotomy a silicone central venous catheter (CVC) with the incidental transthoracic echocardiography (transthoracic echocardiogram, TTE) finding of a tumoral mass of $35 \times 26 \mathrm{~mm}$ in the right atrium (RA), not related with the catheter, which was diagnosed as right atrial myxoma and underwent surgical excision. After reviewing the histopathology probe, the diagnosis of right atrial thrombus was confirmed. In conclusion, differentiating intracardiac right atrial masses (RAMs) could may prove challenging. In our patient, clinical presentation and the preoperative investigations could not differentiate the right atrial thrombus from a myxoma, and only the postoperative histopathology diagnosis was able to guide correct diagnosis.

\section{Introduction}

The presence of a right atrial mass (RAM), despite the advanced diagnostic modalities available, still poses a diagnostic dilemma. RAM constitutes tumors, thrombus, prominent crista terminalis, or endocarditis vegetation (1). Cardiac myxomas are benign and are the most common primary cardiac tumor, $15 \%$ of atrial myxomas arise in the right atrium (RA), and they are usually attached to the interatrial septum (2,3). Myxomatous disease occurs in all age groups, and both sexes have an approximate incidence of 0.5-1.0 cases per million inhabitants per year $(4,5)$.

Incidences of CVC-related right atrial thrombosis (CRAT) are underreported phenomena, but when the thrombus is present, it carries a mortality risk of $18 \%$ in hemodialysis (HD) 
patients and greater than $40 \%$ risk in non-HD patients $(6,7)$. Pathogenic mechanisms incriminated for the development of right atrial thrombosis include mechanical irritation of the myocardial atrial wall in the presence of CVC, emboli entrapped from peripheral venous thrombosis migration, cloths propagation from vena cava, hypercoagulability, and in situ thrombi formation under low-flow atrial conditions $(6,7)$. Presentation of CRAT may be asymptomatic or may be associated with pulmonary embolism, tricuspid valve pathology, leg edema, systemic embolism, infected thrombi, and right heart failure with hemodynamic compromise (6). Fatal complications in CRAT reported are arrhythmias and mechanical cardiac complications (6). The risk of right thrombus formation unrelated with CVC is increased in patients who have antiphospholipid syndrome and who show positive test results for lupus anticoagulant activity or have medium or high levels of anticardiolipin antibodies (3). CRAT may be suspected if there is resistance in blood flow noted during HD.

Transthoracic echocardiography (TTE), transesophageal echocardiography (TEE), and cardiac magnetic resonance (MRI) with gadolinium contrast material can be useful tools for diagnosis and tissue characterization; tumor does not show gadolinium contrast enhancement. TEE has better sensitivity and specificity than TTE; if doubt remains after the echocardiographic examination, cardiac MRI imaging remains a good alternative that can prevent misdiagnosis and redundant invasive measures. MRI has high sensitivity and specificity for the detection of intracardiac mass and can differentiate thrombus, lipoma, inflammatory masses, and many solid tumors. When cardiac myxomas are confirmed and surgically resected, they lose their potential to cause complications, and patients, except for rare cases of recurrences (8), are successfully treated. When CRAT was diagnosed, anticoagulation was the treatment of choice, but when the clot's evolution became unfavorable, surgery remained the 'gold standard' treatment.

In this study, we present the case of a 63-year-old man with chronic kidney disease, in HD, who underwent TTE and identification of a tumoral mass of $35 \times 26 \mathrm{~mm}$ in the RA.

\section{Case report}

Patient. A 64-year-old male with a past medical history of hypertension, end-stage renal disease due to autosomal dominant polycystic kidney disease in HD, type 2 diabetes mellitus insulin-dependent, and paroxysmal atrial fibrillation was referred to the Department of Cardiovascular Surgery, 'Prof. Dr. C. C.' Iliescu Emergency Institute for Cardiovascular Diseases, Bucharest for intermittent chest pain and shortness of breath worsening over the last two weeks, especially on exertion. The chest pain was described as retrosternal and non-radiating. The patient had a long-term HD catheter placed in the right internal jugular vein and left brachial-basilic arteriovenous fistula (BBAVF). HD center staff confirmed maintaining catheter care and heparin usage for thrombosis prevention. No history of thrombosis or catheter infection was documented or described by the patient.
On admission, the patient was clinically stable with no fever, soft abdomen, no peripheral edema, normotensive with blood pressure 120/60 $\mathrm{mmHg}$. Respiratory rate was $22 \mathrm{breaths} / \mathrm{min}$ with an oxygen saturation of $95 \%$. An electrocardiogram (ECG) revealed sinus rhythm with a heart rate of $80 \mathrm{bpm}$. The chest X-ray was normal. Laboratory tests revealed a mild normochromic, normocytic anemia (Hb $11.7 \mathrm{~g} / \mathrm{dl})$, hyperkalemia $(6.2 \mathrm{mmol} / \mathrm{l})$, and high creatinine levels $(12.31 \mathrm{mg} / \mathrm{dl})$. Troponin levels were normal. No microorganisms were found in the patient's blood cultures. Two-dimensional transthoracic echocardiography (TTE) performed showed normal-sized left ventricle (LV), with an ejection fraction of $60 \%$, and a mild left and right atrial enlargement $(\mathrm{LA}=42 \mathrm{~mm}, \mathrm{RA}=43 \mathrm{~mm})$. The right ventricle was dilated with an end-diastolic diameter of $41 \mathrm{~mm}$. No valvular disease was found. Mild hypertrophy of the interventricular septum was present. The most important finding revealed by the TTE was a right atrial echogenic mass of 17/18 mm, independent of the catheter tip (Fig. 1). Coronary angiography revealed normal coronary arteries. No vascularization of the RAM was identified. In view of these findings, we considered the RAM to be either a myxoma or a thrombus. Considering the size of the mass and the high risk of pulmonary embolism, the treatment with anticoagulant was deemed too risky at the time; thus, surgical treatment was elected.

Method. A discussion was held with the patient regarding the risks and benefits involved with the surgical removal of the mass. The patient agreed to the surgical procedure. The patient underwent open-heart surgery for the removal of the RAM. After initiation of cardiopulmonary bypass (CPB), right atriotomy was performed, the tip of the HD catheter and the mass were removed and sent for pathology exam (Fig. 2). The mass was anchored in close proximity to the coronary sinus venosus orifice (Fig. 3). The next day, the rest of the right internal jugular vein catheter was removed, and an HD catheter was inserted in the left femoral vein. Postoperatively the patient experienced self-remitting atrial fibrillation and left upper limb edema for which unfractionated heparin infusion was initiated with good results. Subsequently, anticoagulant therapy (Acenocoumarol) was initiated.

Postoperative TTE showed a free RA. In the six days after surgery, the patient's lab tests revealed leukocytosis $\left(15 \times 10^{3} / \mathrm{mmc}\right)$ and a CPR (C-reactive protein) of $199.2 \mathrm{mg} / 1$. Antibiotics were started (Ceftazidime and Levofloxacin) with the remission of the inflammatory syndrome and a clinically stable patient. After consultation with the nephrology department, it was decided that after every HD session $1 \mathrm{~g}$ of Ceftazidime and $250 \mathrm{mg}$ of Levofloxacin would be administrated. The patient was discharged on the 14th postoperative day. After three weeks, the pathology exam confirmed that the RAM was a recent clot with variable amounts of fibrin (Fig. 4). Three months later, a new long-term HD catheter was placed in the left internal jugular vein, followed by removal of the left femoral catheter. Control TEE was performed at 3 and 6 months, which revealed a free RA. The patient was symptom-free and was kept on anticoagulation treatment with Acenocoumarol. 


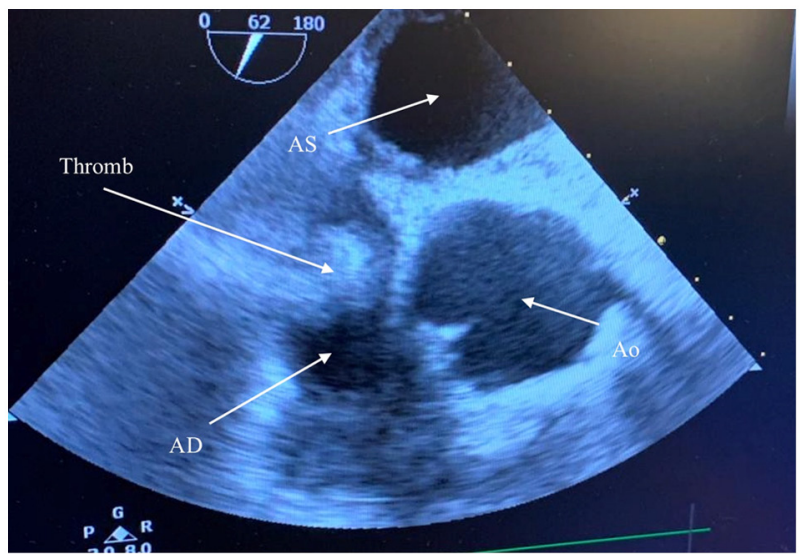

Figure 1.TTE examination revealed a right atrial echogenic mass of 17/18 mm, independent of the catheter tip. TTE, transthoracic echocardiogram.

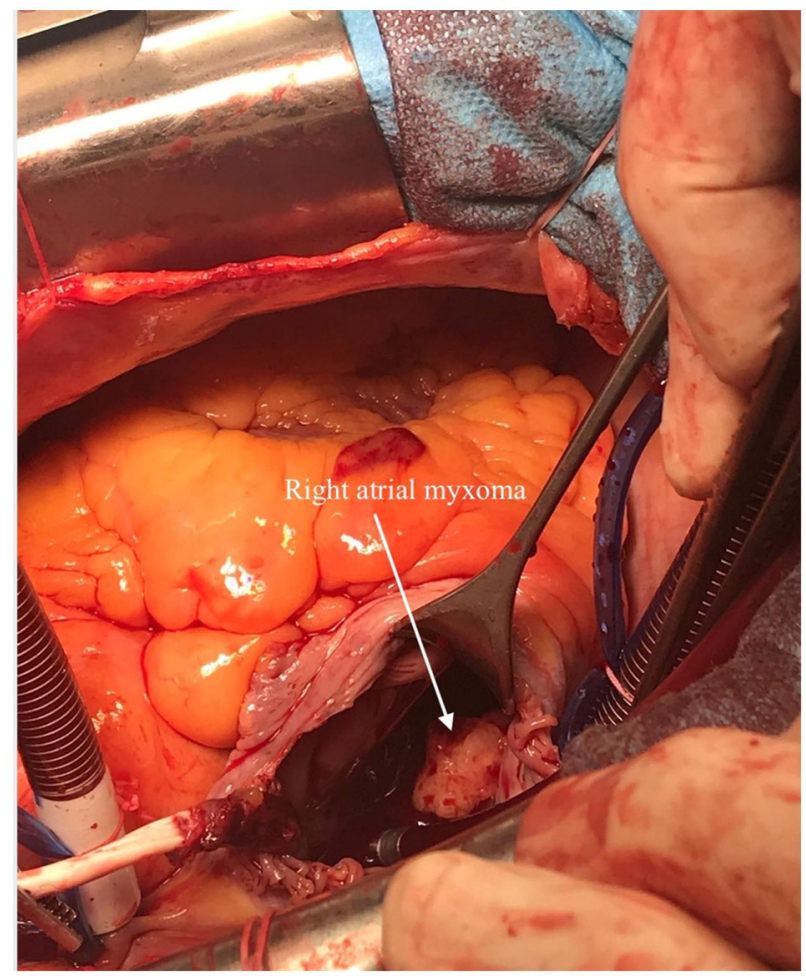

Figure 2. Intraoperator view-right atriotomy approach revealed atrial mass located lateral to the coronary sinus venosus orifice with no related with catheter tip.

\section{Discussion}

The differential diagnosis of RAMs remains a challenge, despite having new imaging techniques. Cardiac mass detection is based on the use of echocardiography, computed tomography and cardiac MRI, with echocardiography being the primary choice $(1,9)$. Right atrial myxomas can be hard to differentiate from right atrial thrombus (10), as was evident in this case report. The risk of intracardiac thrombosis is increased for patients who have a history of atrial fibrillation or indwelling catheters in the RA $(11,12)$. Echocardiographic features, used to differentiate atrial myxoma from thrombus, are represented by an irregular shape of the thrombus,

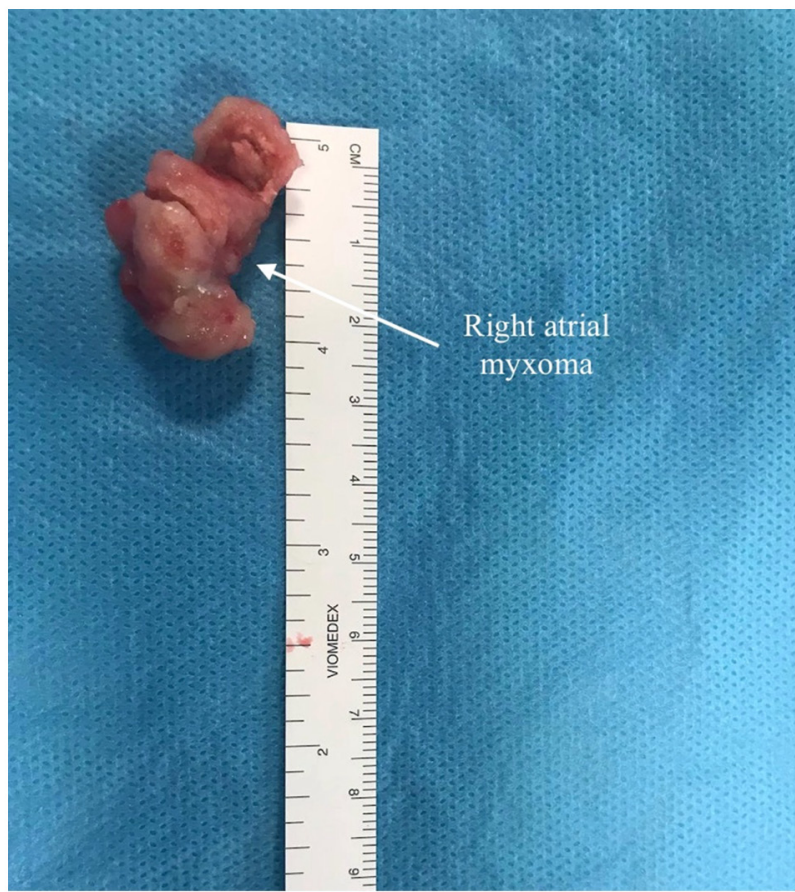

Figure 3. On gross pathological specimen, the right atrial mass was an ovoid, firm, smooth red-tan to dark red-tan surface $(2.5 \times 1.5 \mathrm{~cm})$.

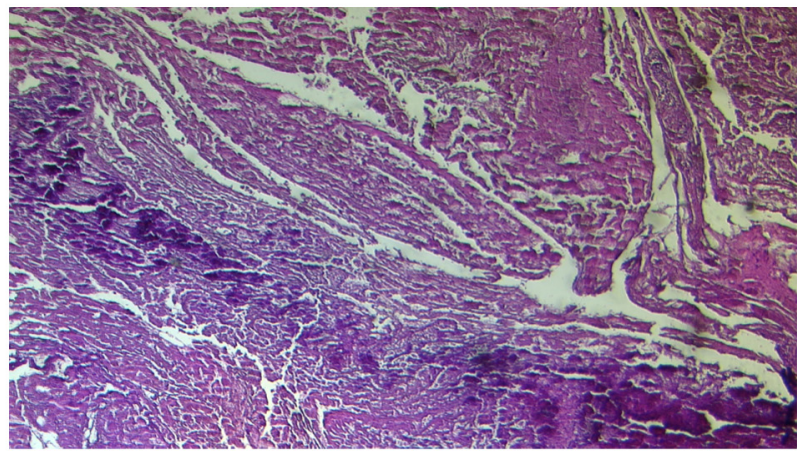

Figure 4. Microscopic views showed focal accumulation of hemosiderin-containing histiocytes, significant deposit of fibrin, and proliferation of the blood vessels and fibroblasts (HE, x40).

immobile mass with a broad base attached to the posterior atrial wall in case of in situ thrombi and a spherical, ovoid mobile mass in case of secondary thrombi. Large mobile thrombi may appear to have no attachment to the atrial wall and they may be mistaken for myxoma $(13,14)$.

CRAT is a rare complication, but it is associated with a high mortality rate. Furthermore, there are no clear guidelines for the treatment of CRAT (6). Thus, it is a decision made on imagistic findings correlated with clinical findings. Right atrial thrombi can develop from venous emboli, they may develop under low-flow conditions, in situ (3) or from around foreign bodies in the right heart (6). In this case, the patient had no history of thrombosis, he had documented heparin usage at HD and no primary cardiac disease was identified on the echocardiography which could lead to cardiac thrombus formation. These findings made the diagnosis even more challenging. Subsequently, it was of the utmost importance to correlate the 
imagistic and clinical findings, to establish a therapeutic attitude. The size of the mass and the risk of pulmonary embolism are important markers that favor the surgical procedure. In case of a thrombus, Hussain et al postulated that clot dimensions have a negative influence on the medical treatment (6). It remains unclear which is the best therapeutic approach, in particular for patients on HD for whom anticoagulation can be challenging. According to Elliott et al warfarin in these patients is linked with a high bleeding risk. A systematic review with 177 patients reported that all individuals that did not receive any treatment, died (15). An important aspect is related to the removal of the atrial catheter, in particular, the timing of extraction. In this patient it was deemed appropriate to remove it in a two-stage procedure and by doing so, minimizing the risks of thrombus displacement. Differentiating RAM still poses difficulties, especially in patients with CVC, and no history of thrombosis, in whom echocardiographic and clinical findings do not support a certain diagnosis. CRAT should always be considered in patients with a RAM and long-term CVC. According to the abovementioned, we consider that surgical removal of big atrial masses, is the preferred treatment.

The diagnosis of a RAM can pose a dilemma despite all the investigations available. In this case, preoperative investigations could not distinguish between myxoma and thrombus, and the exact diagnosis was made postoperatively. Right atrial thrombus should be taken into consideration in the differential diagnosis of RAM, particularly in the presence of a CVC and thrombosis risk factors and in the absence of surgical contraindications should undergo surgical resection.

\section{Acknowledgements}

Not applicable.

\section{Funding}

No funding was received.

\section{Availability of data and materials}

The datasets used and/or analyzed during the current study are available from the corresponding author on reasonable request.

\section{Authors' contributions}

OS, RD, RCG, VAI performed surgical procedures. AT, AC, LR, NB, and ABS reviewed literature data. AT, LR, CD, and $\mathrm{CS}$ conducted the preoperative investigation of the patient. IB, NB, and OS prepared the draft of the article. VAI was the advisor for the surgical procedures. OS, NB, and VAI reviewed the final version of the article. All authors have read and approved the final version of the article.

\section{Patient consent for publication}

Obtained and signed by the patient on 03.02.2020.

\section{Competing interests}

The authors declare that they have no competing interests.

\section{References}

1. Wang J, Wang G, Bi X, Zhang R and Liu C: An unusual presentation of prominent crista terminalis mimicking a right atrial mass: A case report. BMC Cardiovasc Disord 18: 210, 2018.

2. McAllister HA Jr, Hall RJ and Cooley DA: Tumors of the heart and pericardium. Curr Probl Cardiol 24: 57-116, 1999.

3. Cianciulli TF, Saccheri MC, Redruello HJ, Cosarinsky LA, Celano L, Trila CS, Parisi CE and Prezioso HA: Right atrial thrombus mimicking myxoma with pulmonary embolism in a patient with systemic lupus erythematosus and secondary antiphospholipid syndrome. Tex Heart Inst J 35: 454-457, 2008.

4. Iliescu VA, Dorobantu LF, Stiru O, Iosifescu AG, Coman I, Marin S and Filipescu D: Second recurrence of cardiac myxoma 7 years after the initial operation. Chirurgia (Bucur) 103: 239-241, 2008 (In Romanian).

5. Gosev I, Paic F, Duric Z, Gosev M, Ivcevic S, Jakus FB and Biocina B: Cardiac myxoma the great imitators: Comprehensive histopathological and molecular approach. Int J Cardiol 164: 7-20, 2013

6. Hussain N, Shattuck PE, Senussi MH, Velasquez Kho E, Mohammedabdul M, Sanghavi DK, Mustafa U, Balavenkataraman A and Obradovic DM: Large right atrial thrombus associated with central venous catheter requiring open heart surgery. Case Rep Med 2012: 501303, 2012.

7. van Laecke S, Dhondt A, de Sutter J and Vanholder R: Right atrial thrombus in an asymptomatic hemodialysis patient with malfunctioning catheter and patent foramen ovale. Hemodial Int 9: 236-240, 2005.

8. Dorobantu LF, Stiru O, Prodea A, Cioranu R, Georgescu A, Filipescu D and Iliescu VA: Unique case of primary malignant fibrous histiocytoma of the right ventricle with moderator band involvement. Heart Surg Forum 14: E245-E248, 2011.

9. Catez E, Tatnga Ngatcha V and Catez S: An unusual right atrial mass: Case report and review of the literature. Acta Cardiol 65: 477-480, 2010.

10. Matsukuma S, Yamaguchi $\mathrm{H}$ and Hamawaki M: Floating and entangled long right atrial thrombus mimicking myxoma. J Echocardiogr 8: 146-147, 2010.

11. Kingdon EJ, Holt SG, Davar J, Pennell D, Baillod RA, Burns A, Sweny P and Davenport A: Atrial thrombus and central venous dialysis catheters. Am J Kidney Dis 38: 631-639, 2001.

12. Zhao C, Sathya B, Nadal Rios R, Arai AE, Brofferio A, Thein SL and Ruhl AP: Catheter-related right atrial thrombus in sickle cell disease. Clin Case Rep 5: 1898-1900, 2017.

13. Nemani L and Killi S: Right atrial thrombus mimicking myxoma. Indian J Cardiovasc Dis Women-WINCARS 2: 86-90, 2017.

14. Yamane Y, Morimoto H, Okubo S, Koshiyama H and Mukai S: Right atrial calcified ball thrombus mimicking a myxoma. Heart Lung Circ 25: e21-e23, 2016.

15. Elliott MJ, Zimmerman D and Holden RM: Warfarin anticoagulation in hemodialysis patients: A systematic review of bleeding rates. Am J Kidney Dis 50: 433-440, 2007. 